\title{
Modification of Nife Layered Double Hydroxide and Photocatalytic Activity
}

\author{
Huan YU, Weiwei WANG *, Pengfei FANG \\ School of Materials Science and Engineering, Shandong University of Technology, Zibo, Shandong 255091, People's \\ Republic of China \\ crossref http://dx.doi.org/10.5755/j01.ms.25.4.19396
}

Received 06 December 2017; accepted 16 July 2018

\begin{abstract}
Modifying materials by ions with multiple valence states is an effective way for enhancing their properties. NiFe layered double hydroxides (NiFe LDHs) were prepared by using a hydrothermal method. Efficient LDH photocatalyst was synthesized by the modification of NiFe LDHs with Fe(III). Electron microscopy, X-ray diffraction, Brunauer-EmmettTeller surface area, photoluminescence spectroscopy, and UV-Vis diffuse reflectance spectroscopy were used to investigate the morphology, crystal structure, and optical properties of the NiFe LDHs. The photocatalytic and adsorption performances were examined in the degradation of methyl orange. NiFe LDHs exhibited higher adsorption property but lower photocatalytic activity. After modification with $\mathrm{Fe}(\mathrm{III})$, the introducing of $\mathrm{Fe}$ ions and recrystallization of LDHs led to the distance decreasing between $\mathrm{NiFe} \mathrm{LDH}$ sheets and exhibit higher photocatalytic performance.

Keywords: layered double hydroxide, modification, photocatalytic activity, adsorption activity.
\end{abstract}

\section{INTRODUCTION}

Layered double hydroxides (LDHs) are a class of layered anionic clays, generally expressed by the formula $\left[\mathrm{M}^{\mathrm{II}}{ }_{1-\mathrm{x}} \mathrm{M}^{\mathrm{III}}(\mathrm{OH})_{2}\right]\left(\mathrm{A}^{\mathrm{n}-}\right)_{\mathrm{x} / \mathrm{n}} \cdot \mathrm{yH}_{2} \mathrm{O} \quad[1]$. Recently many attentions have been focused on LDHs for their applications in environmental remediation, solar energy storage and conversion, owing to their readily exchanged interlayer anions and tuned composition of the brucite-like layers [2]. However, the process always takes a long time (more than $12 \mathrm{~h}$ ) $[3,4]$. The fast recombination rate of the photogenerated electron-hole pairs and the low removal efficient limit their large-scale practical applications $[5,6]$. Therefore, the photodegradation efficiency of LDHs need to further increase.

Modification is one of the common methods for improving their photocatalytic activity. $\mathrm{NiFe}-\mathrm{LDHs}$ were modified through ion exchanging with $\left[\mathrm{M}\left(\mathrm{C}_{2} \mathrm{O}_{4}\right)_{3}\right]^{3-}$ $(\mathrm{M}=\mathrm{Cr}$ and $\mathrm{Rh})$ and exhibited higher magnetic properties [7]. In addition, studies reported that ions with multiple valence states (such as $\mathrm{Co}, \mathrm{Fe}, \mathrm{Cr}$ ) can offer an effective pathway for electron-hole transport [8,9]. For example, the $\mathrm{Co}(\mathrm{II})$ in $\mathrm{CoAl}-\mathrm{LDH}$ s helped to capture $\mathrm{Pd}(\mathrm{II})$ species through an in situ redox reaction. The obtained CoAlLDH/Pd composites had excellent stability and recyclability [10]. The $\mathrm{Cr}^{3+}$ in the $\mathrm{Au} / \mathrm{Cr}$-substituted hydrotalcite could form the $\mathrm{Cr}^{3+}-\mathrm{Cr}^{6+}$ redox cycle and make it an efficient catalyst for aerobic alcohol oxidation [11]. From this viewpoint, the multiple valence states in LDHs may provide a new means of capturing and separating photogenerated electron-hole pairs and thus improving the photocatalytic activity of the LDHs. This paper presents a simple hydrothermal method for preparing $\mathrm{NiFe}$ LDHs and the modification of NiFe LDHs using $\mathrm{Fe}(\mathrm{III})$-citrate complex under water bath heating. NiFe

\footnotetext{
* Corresponding author. Tel.: +86-15689078202; fax: +86-533-2782198.

E-mail address: wangweiwei@sdut.edu.cn (W. Wang)
}

LDHs exhibited excellent adsorption property. The adsorption amount for methyl orange reached $968.5 \mathrm{mg} / \mathrm{g}$ only within $30 \mathrm{~min}$, which much higher than that of reported values [12-14]. After modification, NiFe LDHs showed better photocatalytic activity and the removal rate reached $95.4 \%$ within $30 \mathrm{~min}$. The modification mechanism was investigated, which provides a facile and effective approach for changing the property of LDHs.

\section{EXPERIMENTAL DETAILS}

\subsection{Synthesis and modification of NiFe LDHs}

All chemicals and reagents used in the experiment were analytical grade without further purification.

To prepare NiFe LDHs: $15 \mathrm{mmol}$ of $\mathrm{Ni}\left(\mathrm{NO}_{3}\right)_{2} \cdot 6 \mathrm{H}_{2} \mathrm{O}$, $5 \mathrm{mmol}$ of $\mathrm{Fe}\left(\mathrm{NO}_{3}\right)_{3} \cdot 9 \mathrm{H}_{2} \mathrm{O}, 20 \mathrm{mmol}$ of $\mathrm{NH}_{4} \mathrm{~F}$, and $50 \mathrm{mmol}$ of urea were dissolved in $100 \mathrm{~mL}$ of deionized water under stirring to form a clear solution. The solution was transferred to an autoclave and maintained at $110{ }^{\circ} \mathrm{C}$ for $8 \mathrm{~h}$. After the reaction, the product was separated by centrifugation and washed with deionized water three times. The product was dried at room temperature and labeled as NiFe LDHs. Studies [3, 4] have reported that the initial mole ratio of $\mathrm{M}^{2+}$ to $\mathrm{M}^{3+}$ was $1 \sim 4$. After reaction, the mole ratio of $\mathrm{M}^{2+}$ to $\mathrm{M}^{3+}$ is usually different with that of initial mole ratio. In our experiments, the mole ratio of $\mathrm{Ni} / \mathrm{Fe}$ is chosen to be 3:1.

To modify NiFe LDHs: ferric ammonium citrate $\left(\left(\mathrm{NH}_{4}\right)_{3} \cdot\left[\mathrm{Fe}(\mathrm{Cit})_{2}\right]\right)$ was dissolved in deionized water to form a clear $\mathrm{Fe}(\mathrm{III})$ complex solution $(25 \mathrm{mmol} / \mathrm{L})$. $\mathrm{NiFe}$ LDHs $(1.5 \mathrm{~g})$ was added in the above solution under vigorously stirring to form a uniform suspension. The suspension was heated at $75{ }^{\circ} \mathrm{C}$ for $12 \mathrm{~h}$ under water bath heating. The following process was the same as that of $\mathrm{NiFe}$ LDHs. The product was labeled as NiFe-Fe LDHs. 


\subsection{Characterization}

X-ray powder diffraction (XRD) patterns were recorded using a D8 ADVANCE X-ray diffractometer with $\mathrm{Cu} \mathrm{K}_{\alpha}$ radiation $(\lambda=1.5406 \AA)$. The scanning electron microscopy (SEM) images were recorded on a FEI-Sirion 200 field emission scanning electron microscope. The transmission electron microscopy (TEM) images, selectedarea electron diffraction (SAED) patterns, high-resolution transmission electron microscopy (HRTEM) images, and energy dispersive spectroscopy (EDS) spectra were taken with a FEI-Tecnai G2 field emission transmission electron microscope. The X-ray photoelectron spectra (XPS) were measured with an ESCALAB 250Xi X-ray photoelectron spectrometer with $\mathrm{Al} \mathrm{K}_{\alpha}(1486 \mathrm{eV})$ as the excitation source. The photocatalytic degradation of methyl orange was conducted in an XPA-7 type photochemical reactor (Xujiang electromechanical plant) equipped with a $300 \mathrm{~W}$ medium pressure mercury lamp as UV light source (mean wavelength $365 \mathrm{~nm}$ ). The UV-Vis diffuse reflectance spectra were recorded by Shimadzu UV-3600 spectrophotometer with an integrating sphere. The $\mathrm{N}_{2}$ adsorption/desorption tests were measured by BrunauerEmmett-Teller (BET) measurements using an NOVA2200e surface area analyzer. Photoluminescence (PL) spectra at $385 \mathrm{~nm}$ were investigated using Hitachi F2500 FL spectrophotometer comprising of a Xe lamp as excitation source. Fourier transform infrared (FTIR) spectroscopy was obtained on a Thermo Nicolet 5700. The $\mathrm{Ni}$ and $\mathrm{Fe}$ contents of the LDHs were determined by inductively coupled plasma atomic emission spectroscopy (PE Optima 2100DV ICP-AES) after dissolving a weighed amount of sample with an aqueous $\mathrm{HCl}$ solution. The carbon content was measured on a LECO CS-444 analyzer.

\subsection{Properties measurements}

The photocatalytic activity and adsorption properties of LDHs were measured using methyl orange. Reaction suspensions were prepared by adding LDHs $(20 \mathrm{mg}$ ) in $50 \mathrm{~mL}$ of methyl orange solution $(20 \mathrm{mg} / \mathrm{L})$. The suspensions were ultrasonically treated for $15 \mathrm{~min}$ and then stirred in the dark for $30 \mathrm{~min}$ to ensure adsorptiondesorption equilibrium. To test the photocatalytic performance of the LDHs, the suspensions were irradiated by UV light. To test the adsorption performance of the LDHs, the suspensions were continuously stirred in the dark. Analytical samples were drawn from the reaction suspensions after various reaction times and analyzed using a UV-Vis spectrophotometer at $467 \mathrm{~nm}$.

\section{RESULTS AND DISCUSSION}

The crystal structure was confirmed from X-ray diffraction patterns (XRD, Fig. 1 a). The reflections of (003), (006), (012), (110) were indexed to typical LDHs materials (JCPDS File No. 51-0463). The basal spacing of LDHs could be calculated based on the d-spacing of $(00 \mathrm{~L})$ planes $([\mathrm{d}(003)+2 \mathrm{~d}(006)] / 2)$. According to the XRD pattern, the basal spacing value of $\mathrm{NiFe}$ LDHs was about $0.766 \mathrm{~nm}$, which coincide well with the values for $\mathrm{CO}_{3}{ }^{2-}$ intercalated LDHs materials [15]. After modification, they still remained the phase of LDHs with a slightly lower

degree of crystallinity and the basal spacing $(0.754 \mathrm{~nm})$, which was lower than that of metallic oxalate complex intercalated LDHs $(1.214 \mathrm{~nm})$ [15]. The results suggested that after modification the interlayer ions of LDHs was $\mathrm{CO}_{3}{ }^{2-}$ and no $\mathrm{Fe}$ complex intercalated in the interlayer, owing to no inert gas protection during the preparation and modification.

XPS spectra were used to determine the oxidation state of elements before and after modification. The peaks at $856.2 \mathrm{eV}$ and $873.7 \mathrm{eV}$ are attributed to the $2 \mathrm{p} 3 / 2$ and $2 \mathrm{p} 1 / 2$ spin state of $\mathrm{Ni}(\mathrm{II})$ for LDHs, respectively [16]. The other two peaks at about $880 \mathrm{eV}$ and $862 \mathrm{eV}$ can be assigned to their corresponding shake-up satellite lines (Fig. $1 \mathrm{~b}$ ). The XPS lines for Fe $2 p$ indicate two sharp peaks at $726.1 \mathrm{eV}$ and $712.5 \mathrm{eV}$ corresponding to the $2 \mathrm{p} 3 / 2$ and $2 \mathrm{p} 1 / 2$ spin states of $\mathrm{Fe}(\mathrm{III})$ (Fig. $1 \mathrm{c}$ ), with a peak splitting of $13 \mathrm{eV}$ [17]. After modification, Ni 2p and Fe 2 p slightly shift to lower energy levels within the permitted error $(0.2 \mathrm{eV})$. No peaks for Fe complex $(725.0 \mathrm{eV}$ and $711.5 \mathrm{eV}$ ) was observed, which also indicate that no $\mathrm{Fe}$ complex intercalated into NiFe LDHs. Both samples also show a peak for F1s, which are from $\mathrm{NH}_{4} \mathrm{~F}$ (Fig. $1 \mathrm{~d}$ ). The mole ratio of $\mathrm{Ni} / \mathrm{Fe}$ for $\mathrm{NiFe} \mathrm{LDHs}$ was $1.82: 1$. After the modification, the mole ratio of $\mathrm{Ni} / \mathrm{Fe}$ was $0.89: 1$. The decrease in the mole ratio of $\mathrm{Ni} / \mathrm{Fe}$ after the modification also indicates the successful doping of $\mathrm{Fe}$ ions. On the basis of the results of elemental analysis measurement, the chemical composition of $\mathrm{NiFe}$ LDHs was estimated to be $\left[\mathrm{Ni}_{0.66} \mathrm{Fe}_{0.34}(\mathrm{OH})_{2}\right]\left(\mathrm{CO}_{3}\right)_{0.17} \cdot \mathrm{yH}_{2} \mathrm{O}(\mathrm{Ni}, 38.6 ; \mathrm{Fe}, 18.5 ; \mathrm{C}$, 1.9), which gives a $\mathrm{Ni} / \mathrm{Fe}$ mole ratio of 1.92 . For $\mathrm{NiFe}-\mathrm{Fe}$ LDHs, $\mathrm{Ni} / \mathrm{Fe}$ mole ratio was 0.95 and the chemical composition is $\left[\mathrm{Ni}_{0.49} \mathrm{Fe}_{0.51}(\mathrm{OH})_{2}\right]\left(\mathrm{CO}_{3}\right)_{0.255} \cdot \mathrm{yH}_{2} \mathrm{O}$.

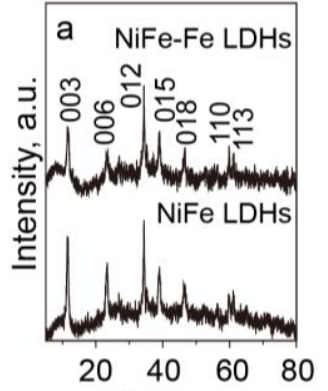

$2 \theta$, degree

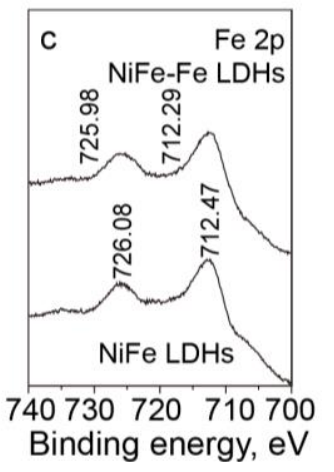

Fig. 1. $\mathrm{a}-\mathrm{XRD}$ patterns of NiFe LDHs and NiFe-Fe LDHs. XPS spectra of NiFe LDHs and NiFe-Fe LDHs; b-Ni 2p; cFe 2p; d-F1s

FTIR spectra (Fig. 2) of both samples provided evidence for the presence of intercalated $\mathrm{CO}_{3}{ }^{2-}$. Both $\mathrm{NiFe}$ 
LDHs and NiFe-Fe LDHs showed the strong peak at $1355 \mathrm{~cm}^{-1}$, which can be attributed to the v3 vibration of $\mathrm{CO}_{3}{ }^{2-}$.

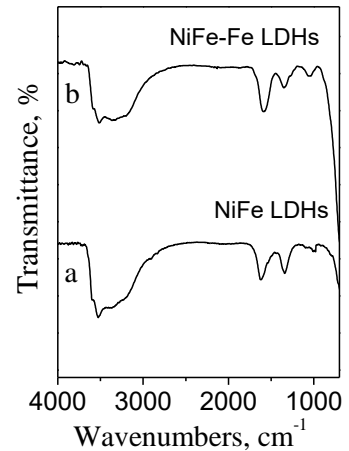

Fig. 2. FTIR spectra: $\mathrm{a}-\mathrm{NiFe} \mathrm{LDHs}$; $b-\mathrm{NiFe}-\mathrm{Fe}$ LDHs

SEM image (Fig. 3 a) of NiFe LDHs at different magnifications show NiFe LDHs nanosheets intersecting and aligned vertically to form a flower-like shape under the orienting function of $\mathrm{NH}_{4} \mathrm{~F}$ [11]. They have a porous structure, and approximately $25 \mathrm{~nm}$ thick. The NiFe LDHs nanosheets are transparent to the electron beam, suggesting that they are very thin (Fig. $3 \mathrm{~b}$ ). The lattice spacing of $0.2658 \mathrm{~nm}$ can be indexed to the (012) plane, which matches the reported values of the hexagonal $\mathrm{LDH}$ structure well (Fig. 3 c) [18]. After the modification, NiFeFe LDHs nanosheets also form the flower-like shape and the nanosheets in the balls are parallel to each other. The distances between these sheets decrease and sheet thickness increases to $200 \mathrm{~nm}$ (Fig. $3 \mathrm{~d}$ ). The lattice spacing of $0.2679 \mathrm{~nm}$ is assigned to the (012) plane of $\mathrm{NiFe}$ LDHs sheets (Fig 3 e and $\mathrm{f}$ ). The SAED pattern on a single sheet reveals the crystalline nature of NiFe-Fe LDHs and can be indexed to the (012) reflection of NiFe LDHs (inset in Fig. $3 \mathrm{f}$ ). The EDS analysis was employed to determine the composition of $\mathrm{NiFe} \mathrm{LDHs}$ and $\mathrm{NiFe}-\mathrm{Fe}$ LDHs, which was similar with the result of XPS analysis. The elemental mapping images also confirm the uniform distribution of $\mathrm{Fe}$ and $\mathrm{Ni}$ elements in $\mathrm{NiFe} \mathrm{LDHs}$ and $\mathrm{NiFe}-\mathrm{Fe}$ LDHs.

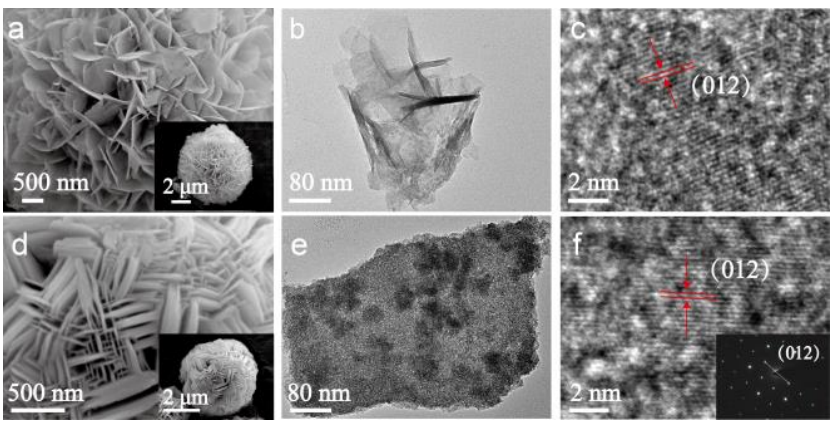

Fig. 3. a, d-SEM images; b, e-TEM images; c, f-HRTEM images of the samples: a-c NiFe LDHs, $d-f$ NiFe-Fe LDHs. The inset of $\mathrm{f}$ is corresponding SAED

In deionized water, citrate ions can form soluble chelate complex with $\mathrm{Fe}^{3+}\left(\left[\mathrm{Fe}(\mathrm{Cit})_{2}\right]^{3-}\right)$, which gradually releases $\mathrm{Fe}^{3+}$ and retards the hydrolysis rate of $\mathrm{Fe}^{3+}$ during the modification process. Therefore, no iron hydroxide or oxide was observed after the modification. $\left[\mathrm{Fe}(\mathrm{Cit})_{2}\right]^{3-}$ was negatively charged and unstable. During the modification,
$\left[\mathrm{Fe}(\mathrm{Cit})_{2}\right]^{3-}$ could absorb on the surface of positive charged $\mathrm{NiFe}$ LDHs layers through electrostatic attraction and decompose with longer reaction time [9]. It results in more $\mathrm{Fe}$ substituted for $\mathrm{Ni}$ in $\mathrm{NiFe} \mathrm{LDHs}$ and an increasing amount of Fe element, consistent with the preceding XPS and XRD analysis.

The photocatalytic activity in methyl orange photodegradation under UV light irradiation was evaluated ( $C_{0}$ and $C_{t}$ are the equilibrium concentrations of methyl orange before and after irradiation, respectively). Before irradiation, NiFe LDHs exhibited better adsorption activity than that of $\mathrm{NiFe}-\mathrm{Fe}$ LDHs. The adsorption rate reached about $87.65 \%$ for NiFe LDHs and only $1.85 \%$ for NiFe$\mathrm{Fe}$ LDHs after stirring in the dark for $30 \mathrm{~min}$ (Fig. $4 \mathrm{a}$ ). After 30 min of irradiation, the degradation rate of methyl orange reached about $95.4 \%$ using the NiFe-Fe LDHs as the photocatalyst (Fig. 4 a). The methyl orange solution was decolorized completely after $60 \mathrm{~min}$ of irradiation and remained unchanged with a long-time irradiation. When using the NiFe LDHs as the photocatalyst, the photodegradation rate of methyl orange was only $4.85 \%$ although the methyl orange removal rate was about $94 \%$ after $120 \mathrm{~min}$ of irradiation (Fig. 4 a). The curve of NiFe LDHs has an upward trend after $30 \mathrm{~min}$ of irradiation, owing to the adsorption/desorption equilibrium changing caused by temperature rising under irradiation.

In order to confirm the photocatalytic activity of NiFeFe LDHs and NiFe LDHs, blank experiments without irradiation were conducted. A blank experiment using $\mathrm{NiFe}-\mathrm{Fe} \mathrm{LDHs}$ as the photocatalyst without irradiation showed that a small quantity of methyl orange $(\sim 3.65 \%)$ was adsorbed after 120 min (Fig. 4 a). However, the adsorption rate of methyl orange for $\mathrm{NiFe}$ LDHs reached $87.35 \%$ after stirred in the dark for $30 \mathrm{~min}$ and $96.85 \%$ continuing to stir for $120 \mathrm{~min}$ (Fig. 4 a). According to the preceding analysis, NiFe LDHs had better adsorption activity and $\mathrm{NiFe}-\mathrm{Fe}$ LDHs exhibited better photocatalytic activity.
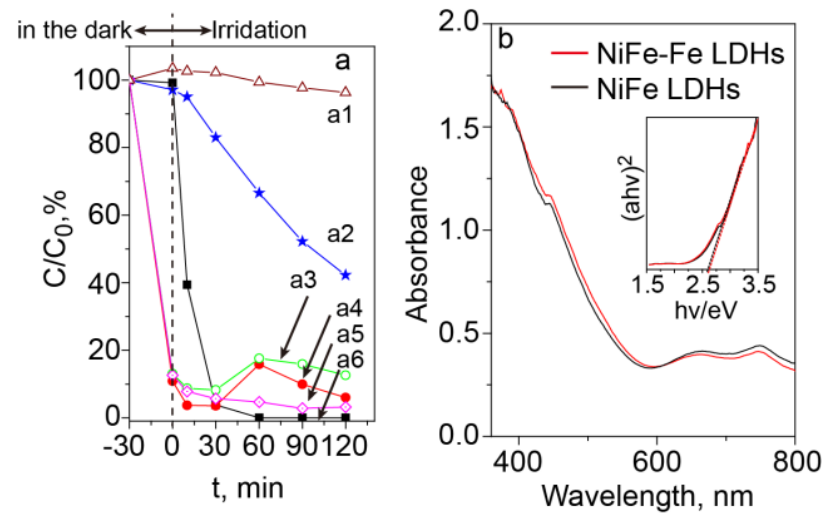

Fig. 4. a-photocatalytic degradation of methyl orange over (a1) blank experiment adding $\mathrm{NiFe}-\mathrm{Fe} \mathrm{LDHs}$ without irradiation, (a2) NiFe LDHs, (a3) blank experiment adding NiFe LDHs without irradiation, and (a4) NiFe-Fe $\mathrm{LDHs} ; \mathrm{b}$ - room temperature UV-Vis diffuse reflectance spectra of NiFe LDHs and NiFe-Fe LDHs. The inset is the plots of $(a h v)^{2}$ vs. photon energy $(h v)$

The maximal methyl orange adsorption removal capacity for $\mathrm{NiFe} \mathrm{LDHs}$ reached $968.5 \mathrm{mg} / \mathrm{g}$, which is much higher than those reported for the anionic dyes 
removal (148 mg/g for MgAl-LDHs [12], $350 \mathrm{mg} / \mathrm{g}$ for $\mathrm{Ni}$ based LDHs [13], and $800 \mathrm{mg} / \mathrm{g}$ for ZnNiAl LDO [14]).

In general, the band gap and specific area of materials are related to their photocatalytic activity for the degradation of pollutants. Fig. $4 \mathrm{~b}$ shows the room temperature UV-Vis diffuse reflectance spectra of $\mathrm{NiFe}$ LDHs and NiFe-Fe LDHs. Both LDHs have absorption in the visible region. The band gap for LDHs was estimated according to the Tauc formula [19] and the plots of $(a h v)^{2}$ versus photon energy $(h v)$ is shown in the inset of Fig. 4 b. The band gap energies are $2.62 \mathrm{eV}$ for $\mathrm{NiFe} \mathrm{LDHs}$ and $2.58 \mathrm{eV}$ for NiFe-Fe LDHs.

Fig. 5 a shows the $\mathrm{N}_{2}$ adsorption/desorption isotherm and pore size distribution curves of $\mathrm{NiFe}$ LDHs and NiFeFe LDHs. Both LDHs are a type IV isotherm with an $\mathrm{H} 3$ hysteresis loop, owing to the aggregation of LDHs nanosheets [20]. A specific surface area of $48.37 \mathrm{~m}^{2} / \mathrm{g}$ with a pore size of $17.06 \mathrm{~nm}$ is observed for NiFe LDHs, which is a little larger than that of NiFe-Fe LDHs $\left(42.82 \mathrm{~m}^{2} / \mathrm{g}\right.$ and $14.27 \mathrm{~nm})$. The pore size is calculated according to the adsorption average pore width (4V/A by BET, where $\mathrm{V}$ represents pore volume, A represents surface area).
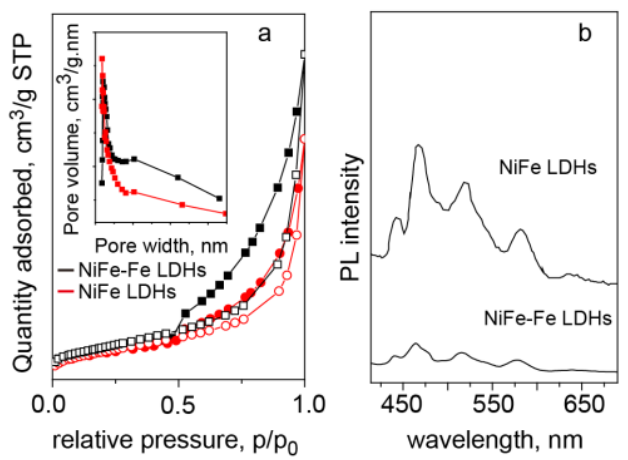

Fig. 5. $\mathrm{a}-\mathrm{N}_{2}$ adsorption/desorption isotherms of NiFe LDHs and $\mathrm{NiFe}-\mathrm{Fe}$ LDHs, Inset: pore size distribution curves;

$\mathrm{b}$-emission spectra at $385 \mathrm{~nm}$ for NiFe-Fe LDHs and $\mathrm{NiFe}$ LDHs

Compared with NiFe LDHs, NiFe-Fe LDHs exhibited higher photocatalytic activity, although they had similar band gaps and specific area. The mainly differences between them were the mole ratio of $\mathrm{Ni}$ to $\mathrm{Fe}$, the thickness of sheet and the distance between sheets. The high recombination rate of photogenerated electron-hole pairs hinders the degradation of methyl orange [21]. The decreasing in distance between NiFe-Fe LDHs sheets could increase particle connectivity and assist in the electron-hole transfer throughout the layered framework, thereby contributing to the high photocatalytic performance [3]. In our experiments, $\mathrm{NiFe}-\mathrm{Fe}$ LDHs with an appropriate network of pores and more $\mathrm{Fe}$ eleme nt could facilitate the efficient transportation of photogenerated charges among the LDHs. Studies also reported that the recombination of photogenerated charges can be investigated through photoluminescence (PL) spectroscopy [22]. For band-band PL emission, the lower the PL intensity is, the higher the separation rate of the photogenerated charges. The emission intensity of NiFe-Fe LDHs was lower than that of NiFe LDHs, and hence, a higher photocatalytic activity was observed (Fig. 5 b).
The adsorption of pollutants by LDHs could be ascribed to two modes: the electrostatic interaction between the LDHs layer and charged adsorbates; the ion exchanging between the interlayer ions and adsorbates. $\mathrm{NiFe}$ LDHs can be regarded as $\mathrm{Ni}(\mathrm{OH})_{2}$ layered structure, in which parts of $\mathrm{Ni}^{2+}$ are replaced by $\mathrm{Fe}^{3+}$, and unequal substitution leads to excess positive charge. The excess positive charges are balanced by intercalation of anions between the layers. The smaller the ratio of $\mathrm{Ni}$ to Fe means the more $\mathrm{Ni}^{2+}$ replaced by $\mathrm{Fe}^{3+}$ and more excessive positive charges in LDHs layers. Meanwhile methyl orange is an anionic organic dye. If electrostatic attraction plays a dominant role, NiFe-Fe LDHs should exhibited better adsorption activity than that of NiFe LDHs. However, $\mathrm{NiFe}$ LDHs exhibited higher adsorption activity, which indicated that the adsorption process was mainly through ion exchanging process. NiFe LDHs have larger interlayer distance, specific area and pore size, which lead to higher adsorption property than that of NiFe-Fe LDHs. Meanwhile the larger distance between NiFe LDHs nanosheets is in favor of the diffusion and adsorption of methyl orange [23]. To investigate the stability, NiFe-Fe LDHs renewability experiment was carried out by repeating photocatalytic degradation of methyl orange. After each cycle, NiFe-Fe LDHs was washed three times by water for the next photocatalytic experiment. The degradation ratio of $\mathrm{NiFe}-\mathrm{Fe} \mathrm{LDHs}$ was maintained at $90 \%$ at $30 \mathrm{~min}$ after four cycle experiments (Fig. 6).

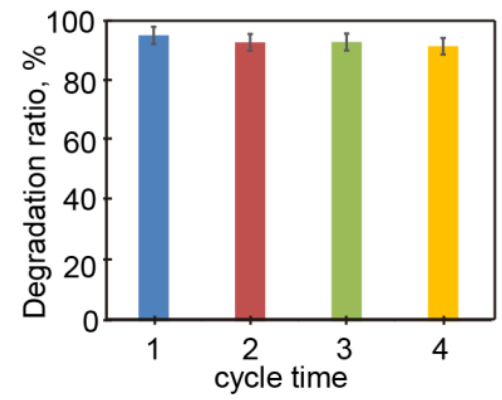

Fig. 6. The regeneration performance of NiFe-Fe LDHs for four cycle experiments

$\mathrm{NiFe}-\mathrm{Fe}$ LDHs before and after four cycle photocatalytic reactions were examined by XRD, SEM and TEM. As shown in Fig. 7, both the fresh and reacted NiFeFe LDHs exhibited a single-phase diffraction pattern assigned to layer structure LDHs, indicating that the original LDHs crystal structure was retained even after four cycles of photocatalytic degradation of methyl orange.

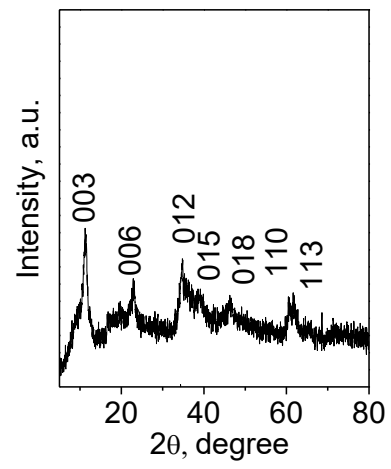

Fig. 7. XRD pattern of NiFe-Fe LDHs after photocatalytic process 
And no noticeable change was observed in the particle morphology of $\mathrm{NiFe}-\mathrm{Fe} \mathrm{LDHs}$ before and after photocatalytic reaction (Fig. 8). NiFe-Fe LDHs exhibited good stability and regeneration capacity for the degradation of methyl orange.

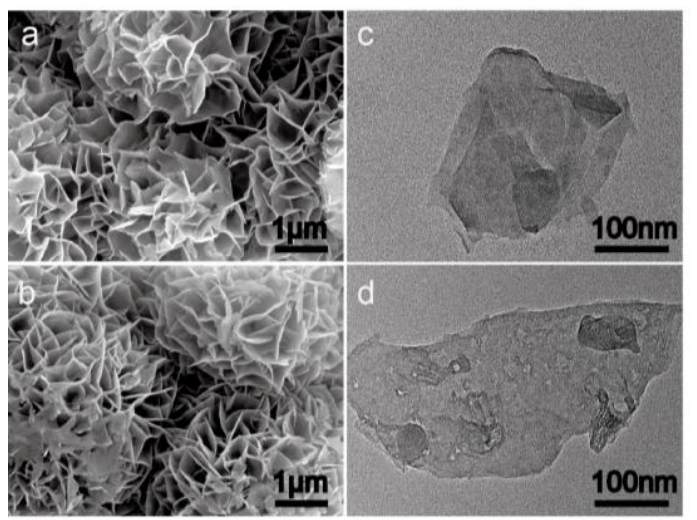

Fig. 8. SEM images of NiFe-Fe LDHs: a-before photocatalytic experiment; $b$-after four cycle photocatalytic experiment. TEM images of NiFe-Fe LDHs; $c$ - before photocatalytic experiment; $\mathrm{d}-$ after four cycle photocatalytic experiment

\section{CONCLUSIONS}

$\mathrm{NiFe}$ LDHs nanosheets with excellent adsorption property were synthesized using a simple hydrothermal method. The larger interlayer distance, specific area and pore size contributed to the higher adsorption activity of $\mathrm{NiFe}$ LDHs. After the modification with negatively charged $\mathrm{Fe}(\mathrm{III})$ complexes, NiFe LDHs displayed better photocatalytic activity in the degradation of methyl orange, owing to the photogenerated carriers having a longer lifetime for participating in photocatalytic reactions.

\section{Acknowledgments}

This work was supported by the Natural Science Foundation of Shandong Provincial (No. ZR2015BM022).

\section{REFERENCES}

1. Yu, J., Wang, Q., O'Hare, D., Sun, L. Preparation of Two Dimensional Layered Double Hydroxide Nanosheets and Their Applications Chemical Society Reviews 46 2017: pp. 5950-5974. http://dx.doi.org/10.1039/C7CS00318H

2. Parida, K., Mohapatra, L., Baliarsingh, N. Effect of $\mathrm{Co}^{2+}$ Substitution in the Framework of Carbonate Intercalated $\mathrm{Cu} / \mathrm{Cr} \mathrm{LDH}$ on Structural, Electronic, Optical, and Photocatalytic Properties The Journal of Physical Chemistry C 116 2010: pp. 22417-22424. http://dx.doi.org/10.1021/jp307353f

3. Lu, Y., Jiang, B., Fang, L., Ling, F., Wu, F., Hu, B. An Investigation of Ultrathin Nickel-iron Layered Double Hydroxide Nanosheets Grown on Nickel Foam for HighPerformance Supercapacitor Electrodes Journal of Alloys and Compounds 714 (20) 2017: pp. 63-70. https://doi.org/10.1016/j.jallcom.2017.04.197

4. Zubair, M., Jarrah, N., Manzar, M., Al-Harthi, M., Daud, M., Mu'Azu, N. Adsorption of Eriochrome Black from Aqueous Phase on MgAl-, CoAl- and NiFe- Calcined Layered Double Hydroxides: Kinetic, Equilibrium and
Thermodynamic Studies 230 2017: pp. 344-352. https://doi.org/10.1016/j.molliq.2017.01.031

5. Li, Y., Bi, H., Shi, X. Simultaneous Adsorption of Heavy Metal and Organic Pollutant onto Citrate-modified Layered Double Hydroxides with Dodecylbenzenesulfonate Environmental Engineering Science 32

2015: pp. 666-675.

https://doi.org/10.1089/ees.2014.0489

6. Qu, J., He, X., Wang, B., Zhong, L., Wan, L., Li, X. Synthesis of Li-Al Layered Double Hydroxides via a Mechanochemical Route Applied Clay Science 120 2016: pp. 24-27. https://doi.org/10.1016/j.clay.2015.11.017

7. Coronado, E., Martí-Gastaldo, C., Navarro-Moratalla, E. Intercalation of $\left[\mathrm{M}(\mathrm{ox})_{3}\right]^{3-},(\mathrm{M}=\mathrm{Cr}, \mathrm{Rh})$ Complexes into $\mathrm{Ni}^{\mathrm{II}} \mathrm{Fe}^{\mathrm{III}}-\mathrm{LDH}$ Applied Clay Science 48 2010: pp. $228-234$. https://doi.org/10.1016/j.clay.2009.11.054

8. Han, J., Dou, Y., Zhao, J., Wei, M., Evans, D., Duan, X. Flexible CoAl LDH@PEDOT Core/shell Nanoplatelet Array for High-performance Energy Storage Small 9

2013: pp. $98-106$. https://doi.org/10.1002/smll.201201336

9. Xu, S., Pan, T., Dou, Y., Yan, H., Zhang, S., Ning, F. Theoretical and Experimental Study on $\mathrm{M}^{\mathrm{II}} \mathrm{M}^{\mathrm{III}}$-Layered Double Hydroxides as Efficient Photocatalysts toward Oxygen Evolution from Water The Journal of Physical Chemistry C 119 2015: pp. 18823-18834. https://doi.org/10.1021/acs.jpcc.5b01819

10. Li, P., Huang, P., Wei, F., Sun, Y., Cao, C., Song, W. Monodispersed Pd Clusters Generated in situ by Their Own Reductive Support for High Activity and Stability in CrossCoupling Reactions Journal of Materials Chemistry A 2 2014: pp. $12739-12745$. http://dx.doi.org/10.1039/C4TA01811G

11. Liu, P., Degirmenci, V., Hensen, E. Unraveling the Synergy Between Gold Nanoparticles and ChromiumHydrotalcites in Aerobic Oxidation of Alcohols Journal of Catalysis 313 2014: pp. 80-91. https://doi.org/10.1016/j.jcat.2014.03.001

12. Ai, L., Zhang, C., Meng, L. Adsorption of Methyl Orange from Aqueous Solution on Hydrothermal Synthesized MgAl Layered Double Hydroxide Journal of Chemical and Engineering Data 56 2011: pp. 4217-4225. https://doi.org/10.1021/je200743u

13. Zaghouane-Boudiaf, H., Boutahala, M., Arab, L. Removal of Methyl Orange from Aqueous Solution by Uncalcined and Calcined MgNiAl Layered Double Hydroxides (LDHs) Chemical Engineering Journal 187 2012: pp. $142-149$. https://doi.org/10.1016/j.cej.2012.01.112

14. Zhang, L., Xiong, Z., Li, L., Burt, R., Zhao, X. Uptake and Degradation of Orange II by Zinc Aluminum Layered Double Oxides Journal of Colloid and Interface Science 469 2016: pp. $224-230$. https://doi.org/10.1016/j.jcis.2016.02.005

15. Parida, K., Sahoo, M., Singha, S. A Novel Approach towards Solvent-free Epoxidation of Cyclohexene by Ti(IV)-Schiff Base Complex-intercalated LDH Using $\mathrm{H}_{2} \mathrm{O}_{2}$ as Oxidant Journal of Catalysis 276 2010: pp. 161-169. https://doi.org/10.1016/j.jcat.2010.09.012

16. Chowdhury, P., Bhattacharyya, K. Ni/Co/Ti Layered Double Hydroxide for Highly Efficient Photocatalytic Degradation of Rhodamine B and Acid Red G: a 
Comparative Study Photochemical \& Photobiological Sciences 16 2017: pp. 835-839.

http://dx.doi.org/10.1039/C7PP00030H

17. Huang, Z., Wu, P., Zhang, X., Wang, X., Zhu, N., Wu, J., Li, P. Intercalation of $\mathrm{Fe}(\mathrm{III})$ Complexes into Layered Double Hydroxides: Synthesis and Structural Preservation Applied Clay Science 65-66 2012: pp. 87-94. https://doi.org/10.1016/j.clay.2012.05.007

18. Tang, D., Liu, J., Wu, X., Liu, R., Han, X., Han, Y., Huang, H., Liu, Y., Kang, Z. Carbon Quantum Dot/NiFe Layered Double-hydroxide Composite as a Highly Efficient Electrocatalyst for Water Oxidation ACS Applied Materials \& Interfaces 6 2014: pp. 7918-7925. https://doi.org/10.1021/am501256x

19. Yun, L., Yang, Z., Yu, Z., Cai, T., Li, Y., Guo, C., Qi, C., Ren, T. Synthesis of Four-angle Star-like CoAl$\mathrm{MMO} / \mathrm{BiVO}_{4}$ p-n Heterojunction and its Application in Photocatalytic Desulfurization RSC Advances 7 2017: pp. $25455-25460$. http://dx.doi.org/10.1039/C7RA03012F

20. Salam, M., Sufian, S., Murugesan, T. Characterization of Nano-crystalline Mg-Ni-Al Hydrotalcite Derived Mixed
Oxides as Hydrogen Adsorbent Materials Chemistry and Physics 142 2013: pp. 213-219.

https://doi.org/10.1016/j.matchemphys.2013.07.008

21. Reszczyńska, J., Grzyb, T., Sobczak, J., Lisowski, W., Gazda, M., Ohtani, B., Zaleska, A. Visible Light Activity of Rare Earth Metal Doped $\left(\mathrm{Er}^{3+}, \mathrm{Yb}^{3+}\right.$, or $\left.\mathrm{Er}^{3+} / \mathrm{Yb}^{3+}\right)$ Titania Photocatalysts Applied Catalysis B: Environmental 163 2015: pp. 40-49. https://doi.org/10.1016/j.apcatb.2014.07.010

22. Baliarsingh, N., Parida, K., Pradhan, G. Effects of Co, Ni, $\mathrm{Cu}$, and $\mathrm{Zn}$ on Photophysical and Photocatalytic Properties of Carbonate Intercalated $\mathrm{MII} / \mathrm{Cr} \mathrm{LDHs}$ for Enhanced Photodegradation of Methyl Orange Industrial \& Engineering Chemistry Research 53 2014: pp. 3834-3841. https://doi.org/10.1021/ie403769b

23. Sadaf, S., Bhatti, H. Batch and Fixed Bed Column Studies for the Removal of Indosol Yellow BG Dye by Peanut Husk Journal of the Taiwan Institute of Chemical Engineers 45 2014: pp. $541-553$.

https://doi.org/10.1016/j.jtice.2013.05.004 